\title{
Circulating tumour cells in breast cancer
}

\author{
Natalia Krawczyk ${ }^{1}$, Malgorzata Banys ${ }^{1}$, Andreas Hartkopf $^{2}$, Carsten Hagenbeck ${ }^{1}$, Carola Melcher $^{1}$ and Tanja Fehm $^{1}$ \\ ${ }^{1}$ Department of Obstetrics and Gynecology, University of Duesseldorf, Moorenstr. 5, 40225 Duesseldorf, Germany \\ ${ }^{2}$ Department of Obstetrics and Gynecology, University of Tuebingen, Calwerstr. 7, 72076 Tuebingen, Germany
}

Correspondence to: Tanja Fehm. Email: tanja.fehm@googlemail.com

\begin{abstract}
Evaluation of isolated tumour cells in bone marrow (BM) and peripheral blood has become a major focus of translational cancer research. The presence of disseminated tumour cells in BM is a common phenomenon observed in $30-40 \%$ of primary breast cancer patients and independently predicts reduced clinical outcome. The detection of circulating tumour cells (CTCs) in blood might become a desired alternative to the invasive and painful BM biopsy. Recent clinical trials confirmed the feasibility of CTC detection as a robust and reproducible parameter for prognostication in both adjuvant and metastatic setting. The characterisation of CTCs might become an important biomarker for therapy monitoring and help to identify specific targets for novel therapeutic strategies.
\end{abstract}

Keywords: breast cancer, disseminated tumour cell, circulating tumour cell, prognosis, biomarkers.

Published: 19/09/2013

Received: $28 / 05 / 2013$

ecancer 2013, 7:352 DOI: 10.3332/ecancer.2013.352

Copyright: (c) the authors; licensee ecancermedicalscience. This is an Open Access article distributed under the terms of the Creative Commons Attribution License (http://creativecommons.org/licenses/by/3.0), which permits unrestricted use, distribution, and reproduction in any medium, provided the original work is properly cited. 


\section{Introduction}

Distant metastasis is the main cause of tumour-related death, but the occult spread of isolated tumour cells (ITCs) in the earliest stage of breast cancer remains undetected by conventional imaging technologies. ITCs in secondary sites, such as blood and bone marrow (BM), are assumed to be precursors of (micro)metastatic disease. The phenomenon of haematogenous dissemination in the metastatic cascade was recognised by several researchers in 19th century [1]. Therefore, detection and characterisation of these cells have become a major focus of translational cancer research. Sensitive assays enable reproducible evaluation of disseminated tumour cells (DTCs) and circulating tumour cells (CTCs) at the single-cell stage.

As demonstrated by a large pooled analysis, the presence of DTCs in BM at the time of diagnosis is associated with reduced survival [2]. In recent years, numerous research groups have endeavoured to replace the invasive and painful BM biopsy with a simple blood test. In the following review, we will discuss the current clinical value of CTCs in the early and advanced breast cancer.

\section{Detection methods}

The low frequency of ITCs, estimated at one tumour cell $/ 10^{7}-10^{8}$ blood cells in patients with advanced cancer, explains the need for extremely sensitive detection assays and tumour cell enrichment [3,4]. Currently, antibody-based and molecular methods are the main techniques for CTC detection.

\section{Tumour cell enrichment}

Three main methods of tumour cell enrichment are currently in use: (a) density gradient centrifugation leads to the separation of mononuclear cells from other blood cells; (b) positive selection leads to the enrichment of CTCs through the use of an antibody targeted against, e.g., cytokeratins (CKs) or epithelial cell adhesion molecule (EpCAM); or (c) negative selection, where the antibody is targeted against a leucocyte antigen (e.g., CD45).

\section{Antibody-based CTC detection}

The majority of translational research trials use antibodies against markers absent from other blood cells; due to the lack of breast cancer-specific antigens, commonly used markers are of epithelial origin (e.g., EpCAM and CKs) [5]. CTCs are then identified by the staining pattern and morphological criteria (consensus recommendations for DTC detection provide a list of phenotypic features that allow a reproducible differentiation between tumour and blood cells) [4]. Antibody-based techniques allow for a direct quantification of detected tumour cells.

\section{Molecular methods}

Molecular methods are mainly based on reverse transcription polymerase chain reaction (RT-PCR) amplification of epithelial or tissuespecific messenger ribonucleic acid (mRNA). Various markers have been established for molecular CTC detection including EpCAM, CKs, and mammaglobin [6]. A limiting factor for RT-PCR-based detection is the illegitimate low-level transcription of targeted mRNA in normal cells and presence of pseudogenes [14]. Therefore, quantitative real-time RT-PCR frequently uses a cut-off value to differentiate between positive and negative findings.

An additional valuable tool for the evaluation of various markers and further characterisation of CTCs with regard to predictive markers, such as human epidermal growth receptor 2 (HER2) or hormone receptor status, is represented by semiquantitative multiplex PCR [7]. 


\section{Commercially available assays}

A variety of commercially available standardised assays for CTC detection has been developed over the last few years. The most commonly used test is the semiautomated, antibody-based, US Food and Drug Administration (FDA)-cleared CellSearch System (Veridex, Warren, New Jersey, United States). This quantitative assay is based on immunofluorescence [8, 9]. After the CTC enrichment by immunomagnetic beads linked with anti-EpCAM antibodies, tumour cells are identified by CK positivity, positive nuclear staining, and CD45 negativity.

One of the most widely used molecular tools for CTC detection is the AdnaTest BreastCancer (AdnaGen AG, Langenhagen, Germany). This tool assay is based on semiquantitative RT-PCR. CTCs are enriched by immunomagnetic beads linked with anti-mucin 1 (MUC1) and anti-EpCAM antibodies and mRNA of three markers gastrointestinal tumour-associated antigen [(GA 733.2), EpCAM, and HER2] is amplified by a multiplex PCR $[7,10,11]$. Both tests have been compared in our previous study and a concordance rate of $70-90 \%$ has been reported.

\section{Detection of CTC in early breast cancer}

Despite its complete surgical extirpation, breast cancer has the ability to recur years after primary diagnosis. As this might happen even in patients without lymph node involvement, occult haematogenous spread of the tumour seems to occur long before the primary tumour becomes clinically detectable. After primary tumour extirpation, tumour cell dissemination leads to minimal residual disease (MRD) and consequently, the aim of successful adjuvant treatment must be its complete eradication. A considerable amount of recent literature on the presence of DTCs in the BM of primary breast cancer patients strongly supports this hypothesis. DTC detection at the time point of diagnosis as well as the detection of persistent DTCs during follow-up is associated with an impaired prognosis and DTCs are currently regarded as an important surrogate of MRD [2, 12-25].

DTC detection is an invasive procedure and associated with increased morbidity. Thus, recent attempts have been made to implement less painful methods that detect CTCs in the peripheral blood (PB) of primary breast cancer patients. However, reports on the prevalence of CTC detection and their prognostic impact are incoherent, which is mainly due to different detection methods and varying cut-off values for positive samples [26-38]. Available literature on the prevalence and prognostic relevance of CTCs, detected in early breast cancer patients, is summarised in Table 1.

In the adjuvant situation, the translational research program of the German SUCCESS-trials prospectively investigated the clinical relevance of CTCs in a large number of primary breast cancer patients. Using the CellSearch System (Veridex), 22\% of the patients presented with $\geq 1$ CTC/7.5-mI PB at primary diagnosis, and CTC detection before taxane-based chemotherapy was an independent predictor of disease-free survival (DFS; HR 1.88) and overall survival (OS; HR 1.91) [28]. Similar results on the prognostic impact of CTCs were reported previously by smaller studies using the CellSearch System or RT-PCR-based techniques for CTC detection (Table 1 and Figure 1) [26, 27, 30-38].

In patients receiving neoadjuvant therapy, monitoring of the primary tumour response allows to evaluate the success of systemic treatment. However, patients might suffer from a relapse despite pathological complete remission. This indicates that systemic response to treatment is independent from the local treatment response of the primary tumour. Accordingly, monitoring of MRD by CTC/DTC detection during neoadjuvant treatment offers the possibility to gain better insights into the influence of systemic treatment on tumour cell dissemination and should also help to optimise treatment strategies.

Interestingly, most of the studies indicate that CTC/DTC detection after neoadjuvant systemic treatment is independent from the primary tumour response and not associated to any clinicopathological characteristics of breast cancer [32, 39-42]. Moreover, in the German GeparQuattro and GeparQuinto trials, the prevalence of CTC detection decreased under neoadjuvant chemotherapy [43, 44]. Mathiesen et al [40] who evaluated the CTC/DTC status before and after neoadjuvant treatment, also found that the number of CTC/DTC-positive patients was decreasing. Whereas, in accordance with other data, DTC detection after neoadjuvant treatment was prognostic of survival, the CTC status had no impact on prognosis. The recently published BEVERLY-2 study also found no prognostic relevance of CTC detection after neoadjuvant treatment [45]. These results seemed to be caused by an increased sensitivity of CTC detection methods as compared with DTC detection. By contrast, Bidard et al [30] and Pierga et al [32] observed a prognostic impact of CTCs detected before and after therapy for early relapse. Similarly, Rack et al presented data of persistent CTC from the adjuvant SUCCESS trials. The persistence of $\geq 1$ CTC after chemotherapy was associated with decreased DFS and the persistence of $\geq 5$ CTCs was associated with decreased OS [29]. 
Table 1. Prognostic relevance of CTC detection in primary breast cancer patients.

\begin{tabular}{|c|c|c|c|c|c|c|}
\hline Author & Year & $N$ & Method & Positivity rate (\%) & Follow-up (months) & Prognostic relevance \\
\hline Franken et al [26] & 2012 & 404 & CellSearch & $19^{4}$ & 48 & $\mathrm{DFS}^{5}, \mathrm{BCSS}^{6}$ \\
\hline Molloy et al [27] & 2011 & 733 & RT-PCR & 8 & 91 & DFS, BCSS \\
\hline Rack et al [28] & 2010 & 2,026 & CellSearch & $22^{1}$ & 35 & DFS, OS \\
\hline Rack et al [29] & 2010 & 1,489 & CellSearch & $9^{4}$ & 32 & $\mathrm{DFS}^{2}, \mathrm{OS}^{1}$ \\
\hline Bidard et al [30] & 2010 & 115 & CellSearch & 23 & 36 & DFS, OS \\
\hline Daskalaki et al [31] & 2009 & 165 & RT-PCR & $55^{1}, 52^{2}$ & 59 & OS $^{1}$ \\
\hline Pierga et al [32] & 2008 & 118 & CellSearch & $23^{1}, 17^{2}$ & 18 & $\mathrm{DFS}^{3}$ \\
\hline Xenidis et al [33] & 2006 & 167 & RT-PCR & 22 & 32 & DFS, OS \\
\hline Benoy et al [34] & 2006 & 116 & RT-PCR & $12-14$ & 26 & None \\
\hline Ntoulia et al [35] & 2006 & 101 & RT-PCR & 14 & 24 & DFS \\
\hline Nieto et al [36] & 2004 & 242 & ICC & 7 & 84 & DFS, OS \\
\hline Zach et al [37] & 2002 & 218 & RT-PCR & 2 & $>12$ & DFS \\
\hline Stathopoulou et al [38] & 2002 & 148 & RT-PCR & 30 & 28 & DFS, OS \\
\hline
\end{tabular}

${ }^{1}$ Before chemotherapy.

${ }^{2}$ After chemotherapy.

${ }^{3}$ Combined positivity before and/or after neoadjuvant chemotherapy.

${ }^{4}$ At least one CTC.

${ }^{5}$ Multivariate analysis.

${ }^{6}$ Univariate analysis.

ICC: Immunocytochemistry.

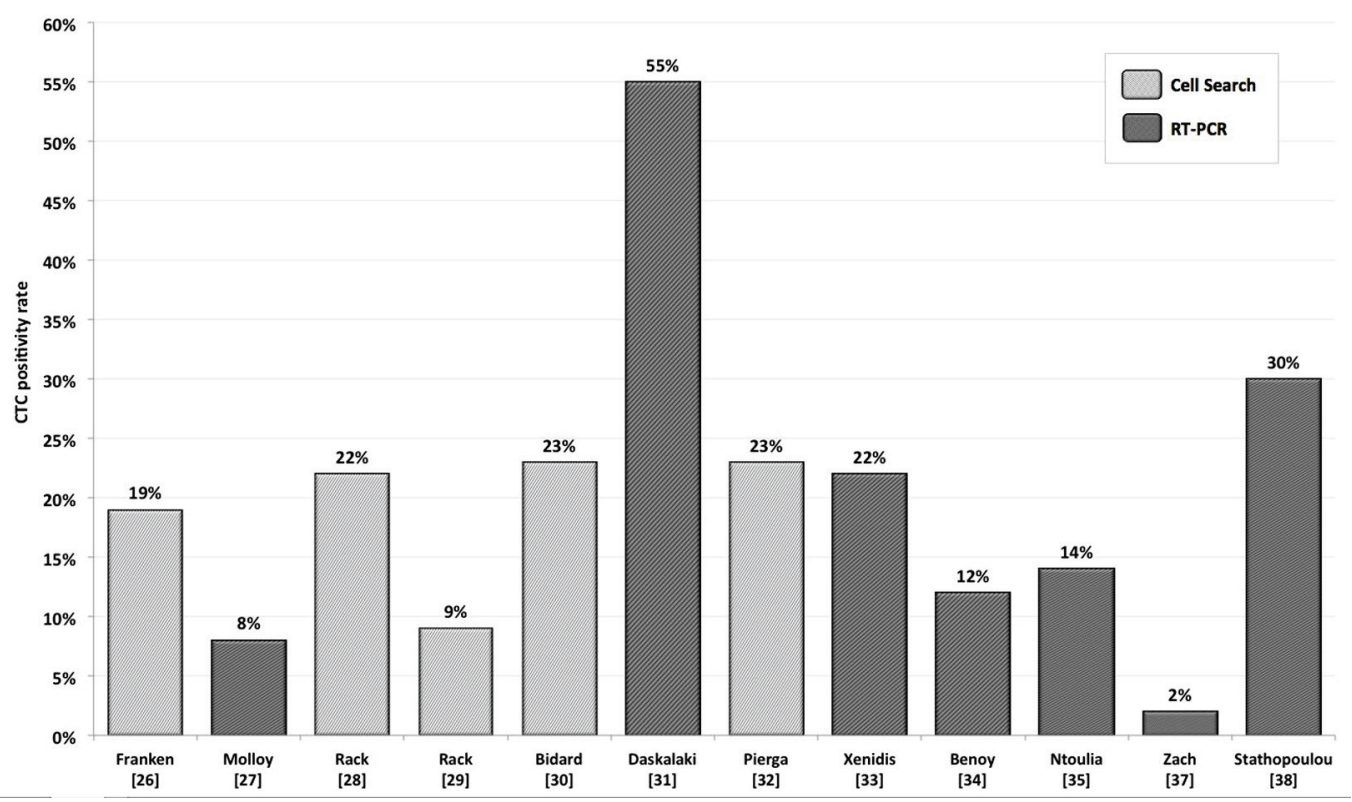

Figure 1. CTC positivity rates depending on detection method reported by several authors. 


\section{Characterisation of CTCs}

ITCs in PB and BM of breast cancer patients are regarded as a surrogate marker for MRD. Therefore, beyond local therapy of primary tumour and lymph node metastases, the eradication of ITC has become a desirable goal of breast cancer treatment. Thus, further characterisation of CTCs, as potential targets for adjuvant therapies, is gaining in importance. However, the choice of systemic treatment is presently based on expression profile of the primary tumour rather than on that of MRD [39].

Phenotypic differences between primary tumour and ITCs in blood and BM have been reported in [7, 46-49]. This phenomenon might be of clinical relevance for treatment decisions concerning endocrine or targeted therapy. Pestrin et al [50] showed a considerable discrepancy in HER2 status in 66 patients with metastatic breast cancer; $29 \%$ of these patients presented with HER2-positive CTCs despite HER2-negative primary tumour, whereas $42 \%$ of patients showed HER2-negative CTCs and HER2-positive primary lesion. In our previous trial, we found HER2-positive DTCs persisting in patients with HER2-negative primary tumour after the completion of adjuvant therapy [48]. Moreover, according to the molecular analyses HER2 gene amplification can be acquired during disease progression [51]. Since HER2-targeted therapy is intended only for patients with HER2-positive primary tumour, HER2-positive MRD in patients with HER2-negative primary lesion remains untreated and may subsequently cause metastasis.

A number of trials have investigated the influence of HER2-targeted therapy on CTCs/DTCs. Bernhard et al [52] transferred autologous HER2-specific T-lymphocytes to a patient with HER2-positive metastatic breast cancer. This experimental treatment was able to eliminate HER2-positive DTCs from the BM, but did not influence the growth of solid metastases. In an interventional study by Rack et al, ten primary breast cancer patients with persistent HER2-positive DTCs received trastuzumab therapy for 12 months. DTC status was then revaluated by follow-up BM biopsies at regular time intervals [53]. HER2-positive DTCs were eradicated in all the patients. However, clinical significance of MRD elimination remains yet unclear. Two randomised clinical trials, DETECT III and TREAT CTC, have been initiated recently to evaluate whether patients with persistent ITCs benefit from HER2-targeted therapy based on HER2 status of their CTCs [54, 55].

Phenotypic discrepancies between primary tumour and MRD have been reported with regard to hormone receptor status as well. A number of studies have shown that CTCs/DTCs are generally hormone receptor negative in spite of hormone receptor positive primary tumour [47, 56, 57]. Previously, we evaluated ER status of DTCs in 107 primary breast cancer patients. Only 12 of 88 patients (14\%) with ER-positive primary tumour presented with ER-positive DTCs in BM while the majority (86\%) had ER-negative DTCs [49]. The discrepancy in ER status between primary tumour and MRD may explain the failure of endocrine therapy in a subset of ER-positive patients. Figure 2 shows the heterogeneity of ITCs.

\section{CTC detection in metastatic breast cancer}

According to a number of studies, $40-80 \%$ of metastatic breast cancer patients present with CTCs in PB. Above the cut-off value of $\geq 5$ CTCs/7.5-ml PB, a patient is considered CTC positive [8, 9]. The most common tool used for CTC detection in advanced breast cancer is the FDA-approved CellSearch System.
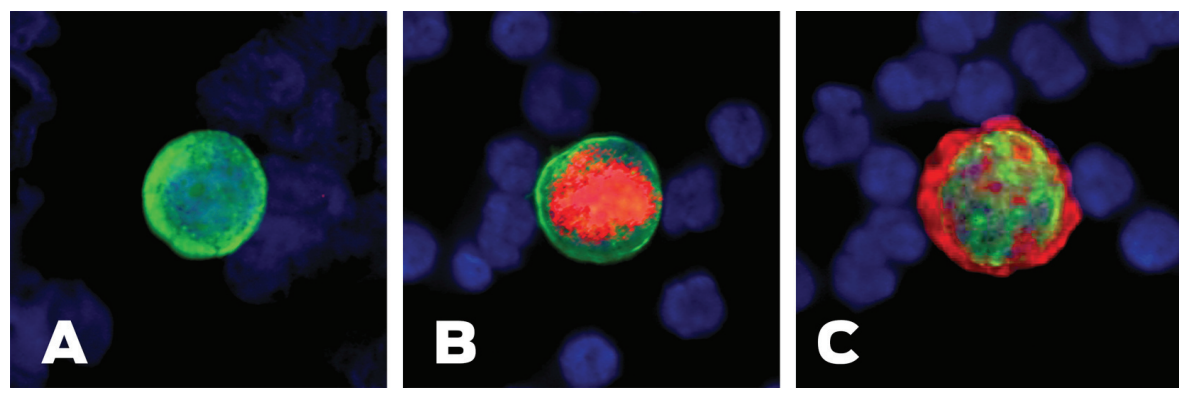

Figure 2. Heterogeneity of ITCs shown by immunofluorescence. (A) Cytokeratin positive ITC. (B) Cytokeratin and ER positive ITC. (C) Cytokeratin and HER2-positive ITC. 


\section{Prognostic value of CTCs in advanced breast cancer}

Prognostic significance of CTCs in metastatic breast cancer has been demonstrated in a number of clinical trials to date (Table 2). The first study to investigate the impact of CTCs on clinical outcome and select an optimal cut-off point for CTC count in this collective of patients was published in [8]. In this multicentre prospective trial, metastatic breast cancer patients with at least five CTCs/7.5-ml PB had significantly shorter progression-free survival (PFS) and OS compared to patients with <5 CTCs/7.5 ml (median PFS: 2.7 versus seven months and OS: 10.1 versus 18 months) [8]. Thus, the cut-off of $\geq 5$ CTCs/7.5-ml PB is used to distinguish between patients with good or poor clinical outcome.

In [58], CTC levels in the same patients cohort were subsequently evaluated at additional time points: before the start of treatment, at 3-5, $6-8,9-15$, and 15-20 weeks of follow-up. Interestingly, the prognostic power of the cut-off value of $\geq 5$ CTCs/7.5-ml PB with regard to PFS and OS remained unchanged. Moreover, the authors observed dynamic changes in CTCs during therapy; the decrease in CTC levels from at least 5/7.5-ml PB to <5/7.5-ml PB during the treatment was associated with a better PFS and OS compared with persistent high CTC counts.

Furthermore, the prognostic value of CTC detection has been recently investigated with regards to molecular subtypes of breast cancer. According to [59], CTCs have no predictive impact on the survival in patient with metastatic HER2-positive breast cancer treated with HER2targeted therapy, in contrast to all other subtypes of breast cancer. This effect might be due to selective effectiveness of HER2-directed therapy against CTCs. These results are concordant with those reported in [60]; in their prospective trial, the authors showed stronger decrease in CTC counts in patients who received targeted therapy (trastuzumab or bevacizumab) in addition to first-line chemotherapy. In contrast, a large prospective multicentre study in [61] reported strong independent prognostic impact of CTCs in metastatic breast cancer patients independent of molecular subtype.

Table 2. Prognostic relevance of CTC detection in metastatic breast cancer patients.

\begin{tabular}{|c|c|c|c|c|c|}
\hline Author & Year & $\begin{array}{l}\text { Number of } \\
\text { patients }\end{array}$ & Method & $\begin{array}{l}\text { Positivity rate } \\
(\%)\end{array}$ & $\begin{array}{l}\text { Prognostic } \\
\text { relevance }\end{array}$ \\
\hline Wallwiener et al [61] & 2013 & 486 & CellSearch & 42 & PFS, OS \\
\hline Giordano et al [59] & 2012 & 517 & CellSearch & $40^{1}$ & PFS, OS \\
\hline Pierga et al [60] & 2012 & 267 & CellSearch & $44^{1}$ & PFS, OS \\
\hline Müller et al [67] & 2012 & 254 & $\begin{array}{l}\text { CellSearch } \\
\text { AdnaTest }\end{array}$ & $\begin{array}{l}\text { CSS: } 50^{1} \\
\text { AT: } 40\end{array}$ & $\begin{array}{l}\text { CellSearch: OS } \\
\text { AdnaTest: none }\end{array}$ \\
\hline Giuliano et al [62] & 2011 & 235 & CellSearch & $40^{1}$ & PFS, OS \\
\hline Reinholz et al [68] & 2011 & 86 & RT-PCR & $\begin{array}{l}56-75^{2} \\
23-38^{3}\end{array}$ & $\begin{array}{l}\text { OS }^{2} \\
\text { None }^{3}\end{array}$ \\
\hline Nakamura et al [69] & 2010 & 107 & CellSearch & $37^{1}$ & PFS \\
\hline Liu et al [70] & 2009 & 74 & CellSearch & n.s. & PFS \\
\hline Tewes et al [71] & 2009 & 42 & AdnaTest & 52 & OS \\
\hline Bidard et al [72] & 2008 & 37 & ICC & 41 & OS \\
\hline Nole et al [73] & 2008 & 80 & CellSearch & 61 & PFS \\
\hline Hayes et al [58] & 2006 & 177 & CellSearch & 54 & PFS, $\mathrm{OS}^{4}$ \\
\hline Budd et al [63] & 2006 & 138 & CellSearch & 43 & OS \\
\hline Benoy et al [34] & 2006 & 32 & RT-PCR & $25-40$ & None \\
\hline Cristofanilli et al [8] & 2004 & 177 & CellSearch & 49 & PFS, OS \\
\hline $\begin{array}{l}1 \geq 5 \text { CTCs. } \\
{ }^{2} \text { CK19 mRNA. } \\
{ }^{3} \text { Mammaglobin mRNA } \\
{ }^{4} \text { At any time during pa } \\
\text { n.s.: not specified. }\end{array}$ & the & & & & \\
\hline
\end{tabular}


Clinical significance of high CTC levels in patients with metastatic breast cancer irrespective of location or number of metastatic sites and hormone receptor status or HER2 status of tumour has been confirmed in a large trial in [62] (median PFS 12.0 versus seven months for patients with CTCs $<5$ and $\geq 5$, respectively; $p<0.001$ ). In this retrospective study of 235 metastatic breast cancer patients, the authors investigated a predictive value of CTC levels for efficiency of the different treatment regimens. Interestingly, patients with high $(\geq 5)$ baseline CTC counts showed only marginal survival benefit from first-line endocrine treatment despite positive hormone receptor status of primary tumour or metastatic lesion. Phenotypic discrepancies between primary tumours and ITCs might be one of the possible factors limiting endocrine therapy in this collective of patients. Therefore, alternative treatment approaches (e.g., antineoangin genetic or targeted therapy) should be evaluated for this population.

\section{Therapy monitoring}

Therapy response in metastatic breast cancer patients is presently being assessed by clinical examination, radiological imaging, and levels of tumour markers in PB. These approaches might be insufficient, and thus new reliable tools are necessary to serve as surrogate markers for the efficiency of treatment. Since changes in CTC counts seem to reflect therapy responses as early as after the first cycle of chemotherapy, CTC dynamics in patients with advanced breast cancer may serve as a new therapy monitoring tool.

Budd et al [63] in their prospective multicentre trial compared the predictive impact of CTC detection to standard imaging with regard to treatment response in metastatic breast cancer patients [63]. The median OS of patients with therapy response assessed by radiological imaging and $\geq 5$ CTCs in PB was significantly shorter than that of patients with therapy response by radiological assessment and $<5$ CTCs (15.3 versus 26.9 months; $p=0.0389$ ). Moreover, patients with radiologic progression and $<5$ CTCs in PB had significantly longer OS comparing patients with radiological progression and $\geq 5$ CTCs in PB (19.9 versus 6.4 months; $p=0.0039$ ).

Whether patients with advanced breast cancer benefit from treatment decisions based on CTC dynamics during therapy is yet unclear. In an attempt to answer this question, the SWOG S0500 trial has been initiated by the Southwest Oncology Group (NCT00382018). The aim of this randomised phase-III study is to investigate whether patients with advanced breast cancer and persistently high levels of CTC $(\geq 5 / 7.5 \mathrm{ml}$ of blood) after three weeks of first-line chemotherapy benefit from switching to an alternative chemotherapy regimen versus waiting for clinical evidence of disease progression before the initiation of new treatment [64]. The recruitment was finished in 2012.

\section{CTC assessment as a real-time 'liquid biopsy' in metastatic breast cancer}

Several studies have reported the phenotypic and genotypic discrepancies between primary tumours, ITC in secondary sites and solid metastases [7, 46-49]. Changes in expression profile of breast cancer can be acquired in the course of the disease [51]. Therefore, the characteristics of the primary tumour, especially with regard to hormone receptor status and HER2 status may not reflect the phenotype of metastases. Since repeated tissue sampling of solid metastatic lesions is not feasible, the assessment of tumour status on CTCs by a simple blood draw as a 'liquid biopsy' might represent an adequate alternative [65].

We previously evaluated HER2 status of CTCs in 254 metastatic breast cancer patients at the time of first diagnosis or disease progression using the CellSearch System. In this prospective multicentre trial, we found HER2-positive CTCs in 25 of the 78 patients (32\%) with HER2negative primary tumour [66]. The clinical value of these findings is being addressed in the prospective randomised DETECT III trial [54], which started recruitment in February 2012. This study investigates the efficacy of anti-HER2 treatment with Lapatinib in advanced breast cancer patients with HER2-positive CTCs, but HER2-negative primary tumour. Similarly, recently initiated TREAT CTC trial should evaluate whether breast cancer patients in adjuvant setting benefit from anti-HER2 therapy based on CTC persistence [55].

\section{Conclusions}

The presence of CTCs in PB of breast cancer patients is associated with impaired clinical outcome in both primary and metastatic settings. CTC detection might lead to the establishment of new treatment strategies in early and advanced breast cancer. However, beyond the mere enumeration, further characterisation of CTCs is gaining in importance. New strategies of targeted treatment based on expression profiles of CTCs are being currently investigated in clinical trials, since the phenotype of CTCs may differ from that of primary tumour. 


\section{References}

1. Ashworth TR (1869) A case of cancer in which cells similar to those in tumors were seen in the blood after death Aus Med $J 14$ 146-9

2. Braun S et al (2005) A pooled analysis of bone marrow micrometastasis in breast cancer N Engl J Med 353(8) 793-802 DOI: 10.1056/NEJMoa050434 PMID: 16120859

3. Ross AA et al (1993) Detection and viability of tumor cells in peripheral blood stem cell collections from breast cancer patients using immunocytochemical and clonogenic assay techniques Blood 82(9) 2605-10 PMID: 8219214

4. Fehm T et al (2006) A concept for the standardized detection of disseminated tumor cells in bone marrow from patients with primary breast cancer and its clinical implementation Cancer 107(5) 885-92 DOI: 10.1002/cncr.22076 PMID: 16874814

5. Fehm T et al (2002) Cytogenetic evidence that circulating epithelial cells in patients with carcinoma are malignant Clin Cancer Res 8(7) 2073-84 PMID: 12114406

6. Becker S et al (2009) Evaluation of a RT-PCR based routine screening tool for the detection of disseminated epithelial cells in the bone marrow of breast cancer patients Breast Cancer Res Treat 117(2) 227-33 DOI: 10.1007/s10549-008-0174-3

7. Fehm T et al (2009) Detection and characterization of circulating tumor cells in blood of primary breast cancer patients by RTPCR and comparison to status of bone marrow disseminated cells Breast Cancer Res 11(4) R59 DOI: 10.1186/bcr2349 PMID: 19664291 PMCID: 2750121

8. Cristofanilli M et al (2004) Circulating tumor cells, disease progression, and survival in metastatic breast cancer $N$ Engl $J$ Med 351(8) 781-91 DOI: 10.1056/NEJMoa040766 PMID: 15317891

9. Cristofanilli $\mathrm{M}$ et al (2005) Circulating tumor cells: a novel prognostic factor for newly diagnosed metastatic breast cancer J Clin Oncol 23(7) 1420-30 DOI: 10.1200/JCO.2005.08.140 PMID: 15735118

10. Demel U et al (2004) Detection of tumour cells in the peripheral blood of patients with breast cancer. Development of a new sensitive and specific immunomolecular assay J Exp Clin Cancer Res 23(3) 465-68 PMID: 15595637

11. Lankiewicz S, Rivero BG and Bocher O (2006) Quantitative real-time RT-PCR of disseminated tumor cells in combination with immunomagnetic cell enrichment Mol Biotechnol 34(1) 15-27 DOI: 10.1385/MB:34:1:15 PMID: 16943567

12. Diel IJ et al (1996) Micrometastatic breast cancer cells in bone marrow at primary surgery: prognostic value in comparison with nodal status J Natl Cancer Inst 88(22) 1652-58 DOI: 10.1093/jnci/88.22.1652 PMID: 8931609

13. Gebauer $\mathrm{G}$ et al (2001) Epithelial cells in bone marrow of breast cancer patients at time of primary surgery: clinical outcome during long-term follow-up J Clin Oncol 19(16) 3669-74 PMID: 11504748

14. Mansi JL et al (1999) Outcome of primary-breast-cancer patients with micrometastases: a long-term follow-up study Lancet 354(9174) 197-202 DOI: 10.1016/S0140-6736(98)10175-7 PMID: 10421301

15. Braun S et al (2000) Cytokeratin-positive cells in the bone marrow and survival of patients with stage I, II, or III breast cancer N Engl J Med 342(8) 525-33 DOI: 10.1056/NEJM200002243420801 PMID: 10684910

16. Wiedswang G et al (2003) Detection of isolated tumor cells in bone marrow is an independent prognostic factor in breast cancer J Clin Oncol 21(18) 3469-78 DOI: 10.1200/JCO.2003.02.009 PMID: 12972522

17. Molino A et al (1997) Bone marrow micrometastases in 109 breast cancer patients: correlations with clinical and pathological features and prognosis Breast Cancer Res Treat 42(1) 23-30 DOI: 10.1023/A:1005747711084 PMID: 9116315 
18. Harbeck $\mathrm{N}$ et al (1994) Tumour cell detection in the bone marrow of breast cancer patients at primary therapy: results of a 3-year median follow-up Br J Cancer 69(3) 566-71 DOI: 10.1038/bjc.1994.103 PMID: 7510120 PMCID: 1968873

19. Pierga JY et al (2004) Clinical significance of immunocytochemical detection of tumor cells using digital microscopy in peripheral blood and bone marrow of breast cancer patients Clin Cancer Res 10(4) 1392-400 DOI: 10.1158/1078-0432.CCR0102-03 PMID: 14977842

20. Janni $W$ et al (2005) The persistence of isolated tumor cells in bone marrow from patients with breast carcinoma predicts an increased risk for recurrence Cancer 103(5) 884-91 DOI: 10.1002/cncr.20834 PMID: 15666325

21. Braun S et al (2000) Lack of effect of adjuvant chemotherapy on the elimination of single dormant tumor cells in bone marrow of high-risk breast cancer patients J Clin Oncol 18(1) 80-6 PMID: 10623696

22. Janni W et al (2011) Persistence of disseminated tumor cells in the bone marrow of breast cancer patients predicts increased risk for relapse-a European pooled analysis Clin Cancer Res 17(9) 2967-76 DOI: 10.1158/1078-0432.CCR-10-2515 PMID: $\underline{21415211}$

23. Wiedswang $\mathrm{G}$ et al (2004) Isolated tumor cells in bone marrow three years after diagnosis in disease-free breast cancer patients predict unfavorable clinical outcome Clin Cancer Res 10(16) 5342-48 DOI: 10.1158/1078-0432.CCR-04-0245 PMID: 15328170

24. Becker $S$ et al (2006) Detection of cytokeratin-positive cells in the bone marrow of breast cancer patients undergoing adjuvant therapy Breast Cancer Res Treat 97(1) 91-6 DOI: 10.1007/s10549-005-9095-6

25. Drageset $\mathrm{V}$ et al (2006) Monitoring of disseminated tumor cells in bone marrow in high-risk breast cancer patients treated with high-dose chemotherapy Int J Canc Supp/ 118(11) 2877-81 DOI: 10.1002/ijc.21709

26. Franken $B$ et al (2012) Circulating tumor cells, disease recurrence and survival in newly diagnosed breast cancer Breast Cancer Res 14(5) R133 DOI: 10.1186/bcr3333 PMID: 23088337

27. Molloy TJ et al (2011) The prognostic significance of tumour cell detection in the peripheral blood versus the bone marrow in 733 early-stage breast cancer patients Breast Cancer Res 13(3) R61 DOI: 10.1186/bcr2898 PMID: 21672237 PMCID: $\underline{3218950}$

28. Rack B et al (2010) Prognostic relevance of circulating tumor cells in the peripheral blood of primary breast cancer patients 33rd Annual San Antonio Breast Cancer Symposium S6-5

29. Rack BK et al (2010) Use of circulating tumor cells (CTC) in peripheral blood of breast cancer patients before and after adjuvant chemotherapy to predict risk for relapse: the SUCCESS trial ASCO Annual Meeting $2010 \mathrm{~J} \mathrm{Clin} \mathrm{Oncol} 28$ 15 s(suppl; abstr 1003)

30. Bidard FC et al (2010) Single circulating tumor cell detection and overall survival in nonmetastatic breast cancer Ann Oncol 21(4) 729-33 DOI: 10.1093/annonc/mdp391

31. Daskalaki A et al (2009) Detection of cytokeratin-19 mRNA-positive cells in the peripheral blood and bone marrow of patients with operable breast cancer Br J Cancer 101(4) 589-97 DOI: 10.1038/sj.bjc.6605183 PMID: 19623181 PMCID: 2736820

32. Pierga JY et al (2008) Circulating tumor cell detection predicts early metastatic relapse after neoadjuvant chemotherapy in large operable and locally advanced breast cancer in a phase II randomized trial Clin Cancer Res 14(21) 7004-10 DOI: 10.1158/1078-0432.CCR-08-0030 PMID: 18980996

33. Xenidis $\mathrm{N}$ et al (2006) Predictive and prognostic value of peripheral blood cytokeratin-19 mRNA-positive cells detected by real-time polymerase chain reaction in node-negative breast cancer patients J Clin Oncol 24(23) 3756-62 DOI: 10.1200/

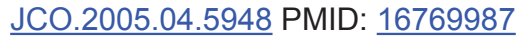


34. Benoy IH et al (2006) Real-time RT-PCR detection of disseminated tumour cells in bone marrow has superior prognostic significance in comparison with circulating tumour cells in patients with breast cancer $\mathrm{Br} J$ Cancer 94(5) 672-80 PMID: 16495933 PMCID: 2361203

35. Ntoulia $\mathrm{M}$ et al (2006) Detection of Mammaglobin A-mRNA-positive circulating tumor cells in peripheral blood of patients with operable breast cancer with nested RT-PCR Clin Biochem 39(9) 879-87 DOI: 10.1016/j.clinbiochem.2006.06.009 PMID: 16925986

36. Nieto Y et al (2004) Prognostic significance of occult tumor cells in the apheresis products of patients with advanced breast cancer receiving high-dose chemotherapy and autologous hematopoietic progenitor cell support Biol Blood Marrow Transplant 10(6) 415-25 DOI: 10.1016/j.bbmt.2004.02.004 PMID: 15148495

37. Zach O et al (2002) Prognostic value of tumour cell detection in peripheral blood of breast cancer patients Acta Med Austriaca Suppl 59 32-4

38. Stathopoulou A et al (2002) Molecular detection of cytokeratin-19-positive cells in the peripheral blood of patients with operable breast cancer: evaluation of their prognostic significance $J$ Clin Oncol 20(16) 3404-12 DOI: 10.1200/JC0.2002.08.135 PMID: 12177100

39. Becker $\mathrm{S}$ et al (2007) Primary systemic therapy does not eradicate disseminated tumor cells in breast cancer patients Breast Cancer Res Treat 106(2) 239-43 DOI: 10.1007/s10549-006-9484-5 PMID: 17260094

40. Mathiesen RR et al (2012) Persistence of disseminated tumor cells after neoadjuvant treatment for locally advanced breast cancer predicts poor survival BCR 14(4) R117 DOI: 10.1186/bcr3242 PMID: $\underline{22889108}$ PMCID: $\underline{3680942}$

41. Hall C et al (2012) Disseminated tumor cells predict survival after neoadjuvant therapy in primary breast cancer Cancer 118(2) 342-8 DOI: $10.1002 / \mathrm{cncr} .26202$

42. Serrano MJ et al (2012) Dynamics of circulating tumor cells in early breast cancer under neoadjuvant therapy Exp Ther Med 4(1) 43-8 PMID: 23060920 PMCID: $\underline{3460281}$

43. von Minckwitz G et al (2010) Capecitabine in addition to anthracycline- and taxane-based neoadjuvant treatment in patients with primary breast cancer: phase III GeparQuattro study J Clin Oncol 28(12) 2015-23 DOI: 10.1200/JC0.2009.23.8303 PMID: $\underline{20308671}$

44. Riethdorf $\mathrm{S}$ et al (2010) Changes in circulating tumor and endothelial cells in peripheral blood of patients treated in the neoadjuvant chemotherapy plus targeted treatment breast cancer study "GeparQuinto" San Antonio Breast Cancer Symposium Abstract PD04-06 Cancer Res 70(Suppl 24) DOI: 10.1158/0008-5472.SABCS10-PD04-06

45. Pierga JY et al (2012) Neoadjuvant bevacizumab, trastuzumab, and chemotherapy for primary inflammatory HER2-positive breast cancer (BEVERLY-2): an open-label, single-arm phase 2 study Lancet Oncol 13(4) 375-84 DOI: 10.1016/S14702045(12)70049-9 PMID: $\underline{22377126}$

46. Solomayer EF et al (2006) Comparison of HER2 status between primary tumor and disseminated tumor cells in primary breast cancer patients Breast Cancer Res Treat 98(2) 179-84 DOI: 10.1007/s10549-005-9147-y PMID: 16552629

47. Banys $M$ et al (2012) The influence of removal of primary tumor on incidence and phenotype of circulating tumor cells in primary breast cancer Breast Cancer Res Treat 132(1) 121-9 DOI: 10.1007/s10549-011-1569-0

48. Krawczyk $\mathrm{N}$ et al (2009) HER2 status on persistent disseminated tumor cells after adjuvant therapy may differ from initial HER2 status on primary tumor Anticancer Res 29(10) 4019-24 PMID: 19846945

49. Fehm T et al (2008) ERalpha-status of disseminated tumour cells in bone marrow of primary breast cancer patients Breast Cancer Res 10(5) R76 DOI: 10.1186/bcr2143 PMID: $\underline{18793387}$ PMCID: $\underline{2614509}$ 
50. Pestrin $\mathrm{M}$ et al (2009) Correlation of HER2 status between primary tumors and corresponding circulating tumor cells in advanced breast cancer patients Breast Cancer Res Treat 118(3) 523-30 DOI: 10.1007/s10549-009-0461-7 PMID: 19597704

51. Meng $S$ et al (2004) HER-2 gene amplification can be acquired as breast cancer progresses Proc Natl Acad Sci U S A 101(25) 9393-98 DOI: 10.1073/pnas.0402993101 PMID: 15194824 PMCID: 438987

52. Bernhard $\mathrm{H}$ et al (2008) Adoptive transfer of autologous, HER2-specific, cytotoxic T lymphocytes for the treatment of HER2overexpressing breast cancer Cancer Immunol Immunother 57(2) 271-80 DOI: 10.1007/s00262-007-0355-7

53. Rack B et al (2012) Trastuzumab clears HER2/neu-positive isolated tumor cells from bone marrow in primary breast cancer patients Arch Gynecol Obstet 285(2) 485-92 DOI: 10.1007/s00404-011-1954-2

54. Bidard FC et al (2013) Clinical application of circulating tumor cells in breast cancer: overview of the current interventional trials Cancer Metastasis Rev 32(1-2) 179-88 DOI: 10.1007/s10555-012-9398-0 PMCID: $\underline{3655223}$

55. Lianidou ES et al (2010) What's new on circulating tumor cells? A meeting report Breast Cancer Res 12(4) 307 DOI:10.1186/ bcr2601 PMID: 20727231 PMCID: 2949631

56. Aktas $\mathrm{B}$ et al (2011) Comparison of estrogen and progesterone receptor status of circulating tumor cells and the primary tumor in metastatic breast cancer patients Gynecol Oncol 122(2) 356-60 DOI: 10.1016/j.ygyno.2011.04.039 PMID: 21605893

57. Ditsch $\mathrm{N}$ et al (2003) Estrogen receptor expression profile of disseminated epithelial tumor cells in bone marrow of breast cancer patients Recent Results Cancer Res 162 141-7 DOI: 10.1007/978-3-642-59349-9 12 PMID: 12790328

58. Hayes DF et al (2006) Circulating tumor cells at each follow-up time point during therapy of metastatic breast cancer patients predict progression-free and overall survival Clin Cancer Res 12(14 Pt 1) 4218-24 DOI: 10.1158/1078-0432.CCR-05-2821 PMID: 16857794

59. Giordano A et al (2012) Circulating tumor cells in immunohistochemical subtypes of metastatic breast cancer: lack of prediction in HER2-positive disease treated with targeted therapy Ann Oncol 23(5) 1144-50 DOI: 10.1093/annonc/mdr434

60. Pierga JY et al (2012) High independent prognostic and predictive value of circulating tumor cells compared with serum tumor markers in a large prospective trial in first-line chemotherapy for metastatic breast cancer patients Ann Oncol 23(3) 618-24

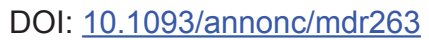

61. Wallwiener $\mathrm{M}$ et al (2013) The prognostic impact of circulating tumor cells in subtypes of metastatic breast cancer Breast Cancer Res Treat 137(2) 503-10 DOI: 10.1007/s10549-012-2382-0

62. Giuliano $\mathrm{M}$ et al (2011) Circulating tumor cells as prognostic and predictive markers in metastatic breast cancer patients receiving first-line systemic treatment Breast Cancer Res 13(3) R67 DOI: 10.1186/bcr2907 PMID: 21699723 PMCID: $\underline{3218956}$

63. Budd GT et al (2006) Circulating tumor cells versus imaging--predicting overall survival in metastatic breast cancer Clin Cancer Res 12(21) 6403-09 DOI: 10.1158/1078-0432.CCR-05-1769 PMID: 17085652

64. Exchange of Ideas and Information on the Issue of Competitive Change in the Electric Power Industry. Topic: What Are the Issues Involved in Competition?, in Committee on Energy and Natural Resources 1997 (Washington, DC: Bureau of National Affairs) p. 17

65. Alix-Panabieres C and K Pantel (2013) Circulating tumor cells: liquid biopsy of cancer Clin Chem 59(1) 110-18 DOI: $10.1373 /$ clinchem.2012.194258

66. Fehm T et al (2010) HER2 status of circulating tumor cells in patients with metastatic breast cancer: a prospective, multicenter trial Breast Cancer Res Treat 124(2) 403-12 DOI: 10.1007/s10549-010-1163-x PMID: 20859679

67. Muller V et al (2012) Prognostic impact of circulating tumor cells assessed with the CellSearch System and AdnaTest Breast in metastatic breast cancer patients: the DETECT study Breast Cancer Res 14(4) R118 DOI: 10.1186/bcr3243 
68. Reinholz MM et al (2011) Cytokeratin-19 and mammaglobin gene expression in circulating tumor cells from metastatic breast cancer patients enrolled in north central cancer treatment group trials, n0234/336/436/437 Clin Cancer Res 17(22) 7183-93 DOI: 10.1158/1078-0432.CCR-11-0981 PMID: 21976532

69. Nakamura S et al (2010) Multi-center study evaluating circulating tumor cells as a surrogate for response to treatment and overall survival in metastatic breast cancer Breast Cancer 17(3) 199-204 DOI: 10.1007/s12282-009-0139-3

70. Liu MC et al (2009) Circulating tumor cells: a useful predictor of treatment efficacy in metastatic breast cancer $J$ Clin Oncol 27(31) 5153-9 DOI: 10.1200/JCO.2008.20.6664 PMID: 19752342

71. Tewes $\mathrm{M}$ et al (2009) Molecular profiling and predictive value of circulating tumor cells in patients with metastatic breast cancer: an option for monitoring response to breast cancer related therapies Breast Cancer Res Treat 115(3) 581-90 DOI: $10.1007 /$ $\underline{\text { s10549-008-0143-x }}$

72. Bidard FC et al (2008) Prognosis of women with stage IV breast cancer depends on detection of circulating tumor cells rather than disseminated tumor cells Ann Oncol 19(3) 496-500 DOI: 10.1093/annonc/mdm507 PMID: 18187488

73. Nole $\mathrm{F}$ et al (2008) Variation of circulating tumor cell levels during treatment of metastatic breast cancer: prognostic and

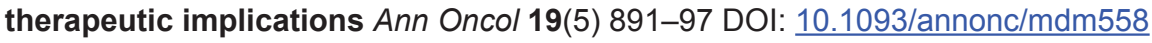

